\title{
Impacto económico de la celebración de un evento deportivo: Campeonato del Mundo de MotoGP en Valencia
}

\author{
Ma LUISA MARTÍ SELVA \\ Grupo de Economía Internacional. Departamento de Economía y Ciencias Sociales, \\ UNIVERSIDAD POLITÉCNICA DE VALENCIA, ESPAÑA.E-mail: mlmarti@esp.upv.es
}

ROSA PUERTAS MEDINA

Grupo de Economía Internacional. Departamento de Economía y Ciencias Sociales, UNIVERSIDAD POLITÉCNICA DE VALENCIA, ESPAÑA. E-mail: rpuertas@esp.upv.es

\begin{abstract}
RESUMEN
En el trabajo que se presenta se analizan los efectos económicos sobre el sector turístico valenciano de un evento deportivo de relevancia internacional como es el Campeonato Mundial de MotoGP. La utilización de la metodología input-output ha permitido diferenciar entre los efectos directos, indirectos e inducidos que se producen sobre el turismo de la provincia donde se realiza la competición deportiva. Así, se concluye que el gasto realizado por los asistentes al Campeonato supone una notable aportación a los salarios, el empleo y el PIB, manifestando así su importancia dentro de la actividad turística valenciana.
\end{abstract}

Palabras clave: Deporte, análisis input-output, impacto turístico.

\section{Economic Impact of a Sporting Event: MotoGP World Champonship in Valencia}

\begin{abstract}
Work presented analyses the economic effects on the Valencian tourism sector of a sporting event of international significance as the World Championship of MotoGP. Using the input-output methodology has allowed to differentiate between the effects direct, indirect and induced that occur on the tourism in the province where is the sporting event. Thus concludes that spending by those attending the Championship is a notable contribution to wages, employment and GDP, thus demonstrating its importance within the Valencian tourism.
\end{abstract}

Keywords: Sport, Input-Output Analysis, Tourist Impact.

Clasificación JEL: L83, D57

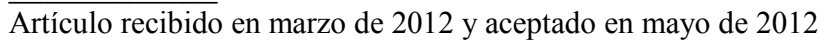

Artículo disponible en versión electrónica en la página www.revista-eea.net, ref. ə-30208 


\section{INTRODUCCIÓN}

La celebración de grandes eventos supone un elemento de desarrollo socioeconómico y una opción estratégica para las ciudades, pudiendo ser utilizada para posicionarse en un contexto internacional. Todo ello permite atraer recursos económicos concretados en la realización de nuevas inversiones, prestación de servicios e incluso en una mejora de la imagen de la ciudad y su entorno. Además, la dinamización y desarrollo del tejido socio-económico del territorio atraerá la celebración de nuevos eventos y mayor afluencia turística (Fomento de San Sebastián, 2005).

En este artículo se analizarán las consecuencias económicas de la celebración del Campeonato Mundial de MotoGP en Valencia. Se trata de la competición de motociclismo más antigua (desde 1949) e importante del mundo celebrándose en 14 países, cuatro continentes y con una cobertura televisiva global. La Competición objeto de estudio es la que tuvo lugar durante noviembre de 2010 en el Circuit Ricardo Tormo ubicado a 20 minutos de la ciudad de Valencia y con unas instalaciones que ofrecen un amplio abanico de posibilidades como presentaciones de escuderías de Fórmula 1, pruebas de atletismo, conciertos, etc. Desde 1999 hasta 2008 el número de asistentes superaba anualmente los 200.000 aficionados, y es a partir de 2009 cuando la cifra empieza a descender hasta llegar a los 80.774 en 2010 . Es importante tener en cuenta que la asistencia a los eventos deportivos es muy sensible a la situación económica y su demanda se ve fuertemente afectada, al no tratarse de bienes de primera necesidad para el consumidor.

El objetivo de la investigación responde a la inquietud que paulatinamente se ha ido extendiendo entre las ciudades y entidades organizadoras de conocer la repercusión de los eventos deportivos en el seno de su economía. La orientación del estudio no está encaminada al gasto directo generado por los propios atletas, sino al gasto del aficionado que acude como espectador al acontecimiento deportivo. Los resultados permitirán evitar la sobrevaloración que en muchas ocasiones se asocia a la celebración de competiciones deportivas internacionales.

La metodología de impacto económico propuesta está basada en el análisis input-output, el cual se fundamenta en la relaciones de interdependencia que existen entre el gasto de los aficionados y las actividades productivas que se ven incrementadas, tratando de determinar el efecto multiplicador que dicha demanda tiene sobre el resto de la economía donde ejerce su influencia. La estimación de los gastos iniciales realizados por los asistentes al evento deportivo puede ser determinado mediante encuestas, sin embargo existe un "efecto arrastre" sobre el resto de la actividad productiva difícilmente cuantificable de forma directa.

En este sentido el análisis input-output facilita el cálculo de todos los efectos asociados a la celebración de este campeonato. El hecho de que los asistentes 
demanden una serie de productos y servicios genera en la economía efectos directos, indirectos e inducidos, necesarios para satisfacer la demanda final realizada en el contexto de la competición.

El artículo se estructura de la siguiente forma. En la sección 2 se hace una revisión bibliográfica sobre la importancia del deporte en la economía. En la sección 3 se explica la metodología del análisis input-output que se aplicará en la parte empírica del trabajo. En la sección 4 se muestran los resultados obtenidos. Y por último, en la sección 5 se resumen las conclusiones del estudio.

\section{REVISIÓN BIBLIOGRÁFICA DEL IMPACTO ECONÓMICO DEL DEPORTE}

El desarrollo del deporte en los últimos años ha desencadenado una serie de cambios que lo configuran no solo como una actividad lúdica y saludable, sino como un hecho social de gran significatividad en nuestras vidas. A su vez, es un potencial para las empresas que buscan en el patrocinio su estrategia de marketing. A principios de los años 90 los investigadores universitarios empezaron a evaluar el impacto económico que el deporte podría representar sobre una determinada región española (Alonso et al, 1991; Martinez del Castillo et al, 1991; Brunet, 1992; Quesada y Diez, 1993).

En este ámbito, los instrumentos que se han venido utilizando para elaborar las magnitudes económicas del deporte son muy variados, principalmente destacan: los modelos de equilibrio general, el método input-output y el análisis coste beneficio ${ }^{1}$. Mientras que los modelos de equilibrio general precisan de una serie de supuestos que condicionarán los resultados, el análisis coste beneficio constituye un método más amplio y flexible facilitando la identificación y evaluación de la repercusión de actos públicos. Por su parte, el análisis input-output va más allá, pues cuantifica de forma precisa la incidencia económica de una modificación de la demanda a través de unos coeficientes técnicos. Incluso, podría ser utilizado para simular la posible celebración de nuevos eventos que justificaría las futuras inversiones.

Sin embargo, como cualquier otra metodología no está exenta de críticas, en ocasiones rebatibles simplemente clarificando el objetivo del análisis y la información disponible para la realización del mismo. Lopez et al (2011) resalta dos condicionantes existentes como son la proporcionalidad y la homogeneidad, que impiden contemplar economías de escala y reducen el grado de precisión de los resultados, respectivamente. En el ámbito de la economía del turismo también se encuentran trabajos que utilizan la metodología input-output, como en el trabajo de Polo y Valle (2011) para analizar la actividad turística en las islas

\footnotetext{
${ }^{1}$ En los trabajos de Pedrosa y Salvador (2003) y Fourie y Santana-Gallego (2011) se encuentra una descripción detallada de dichas metodologías.
} 
Baleares o el artículo de Perles (2006) donde examina el impacto económico a través del análisis input-output que las fiestas populares de Moros y Cristianos generan sobre el destino turístico de Calpe.

Esta línea de investigación se ha seguido desarrollando y pueden encontrarse algunos trabajos que incluyen tanto la repercusión del gasto de los aficionados como de las empresas y clubes relacionadas con el deporte. Así, Espejo-Saavedra y Algarra (2005) platean un modelo de impacto económico del futbol profesional en España a partir de las Tablas Input-Output (TIO), concluyendo que el multiplicador del futbol profesional se aproxima a la media del sector servicios, siendo la energía y la construcción las actividades más influyentes en el crecimiento económico. En el trabajo aplicaremos la metodología input-output puesto que permitirá analizar el impacto del gasto de los asistentes al Campeonato Mundial de MotoGP sobre el entramado turístico fundamentándose en la posibilidad de estimar, de forma sectorial y agregada, los efectos que provocan en las actividades económicas propias del turismo.

A su vez, Maudos (2007) y Fernández et al (2007), utilizando el modelo input-output, analizan el impacto económico de la $32^{\circ}$ America's Cup en Valencia distinguiendo entre el impacto del gasto de los turistas y las inversiones realizadas por la Administración Pública, concluyendo que dicho evento ha supuesto un aumento considerable del PIB y el empleo de la Comunidad Valenciana (CV) durante tres años consecutivos, permitiendo a su vez que la ciudad sea más conocida internacionalmente. Por su parte, Barguet y Gouguet (2008) a través del análisis coste-beneficio calculan el impacto económico de la Copa del Mundo de Rugby 2007 en Francia teniendo en cuenta inversiones, gastos de organización, animación, consumo de los equipos y visitantes. Watson et al (2008) con la técnica input-output realizan un análisis similar aplicado a la "industria del golf" en Colorado.

Un estudio de impacto económico de la celebración de un evento deportivo debe tener en cuenta que el aumento del turismo constituye uno de los efectos más importantes derivados del mismo. Según el Centro de Estudios Económicos Tomillo (CEET, 2005) la capacidad de atracción turística de una ciudad depende de factores muy diversos como: implementación de estrategias, campañas turísticas, imagen de la ciudad, proximidad a las tradicionales fuentes de turismo. Fourie y Santana-Gallego (2011) mediante la aplicación de un modelo de gravedad concluyen que un evento deportivo incrementa aproximadamente un $8 \%$ el turismo en el año de su celebración.

La utilización del modelo input-output en este artículo permite analizar con precisión la incidencia sobre el turismo valenciano del gasto de los asistentes al Campeonato. Respecto a este método, una de las primeras aplicaciones se encuentra en el trabajo de Herderson (1955) donde se utiliza el método input-output para analizar la economía italiana. En el ámbito del transporte marítimo la 
literatura recoge innumerables trabajos desde Hill (1975) en donde se determinó el impacto del puerto de Baltimore (EEUU) hasta Martí et al (2009) que recoge su aplicación a los distintos puertos de la CV. También se ha utilizado esta metodología para determinar los efectos causados por diversas competiciones lúdico-deportivas (Barker et al, 2002; Crompton et al, 2001; Kasimati, 2003). Más recientemente, Collins et al (2009) proponen la utilización del modelo input-output para cuantificar impactos ambientales de eventos deportivos celebrados en Reino Unido. En el ámbito turístico se ha utilizado para analizar el impacto económico de los hoteles en la ciudad de Sevilla y el festival de música Jazz de Umbría (González, 2010 y Bracalente et al, 2011). En nuestro caso, esta herramienta permitirá profundizar en el entramado productivo del turismo, ofreciendo cifras de producción, empleo, impuestos, entre otras, que facilitará conocer la importancia del evento deportivo en la economía valenciana.

La investigación que se presenta solo tendrá en cuenta el gasto del aficionado asistente al campeonato, debido a que las instalaciones llevan funcionando más de una década y a la importancia del turismo en la CV. El objetivo es, por tanto, analizar el impacto del evento sobre el turismo valenciano con la finalidad de evaluar y justificar los esfuerzos privados y gubernamentales para traer la celebración de este evento a la ciudad de Valencia.

\section{METODOLOGÍA}

El análisis input-output es una herramienta idónea para el desarrollo de los estudios cuantitativos sobre cómo las actividades de una infraestructura o un evento influyen en su entorno económico. Se trata de una metodología muy utilizada en la determinación del impacto económico y en la relevancia de las grandes decisiones de inversión en infraestructuras de transporte, sanidad, ocio, o cualquier otro servicio que tenga una compleja relación con el resto de actividades económicas.

La herramienta básica utilizada ha sido la TIO de la provincia de Valencia para el 2010 creada a partir de la TIO de la CV del 2000. La transformación de una TIO a otra se ha realizado a partir de la información de la contabilidad regional y la aplicación del método iterativo RAS (Stone, 1969).

E1 Instituto Valenciano de Estadística pública periódicamente la TIO de la $\mathrm{CV}$ desagregada en 84 sectores. Esta ha sido la fuente inicial de datos para la construcción de la TIO valenciana del turismo. En primer lugar, se han identificado las ramas de actividad relativas al sector turístico, de tal forma que se correspondieran con las de la cuenta satélite del turismo publicada por el INE.

Posteriormente, y debido al desfase temporal entre la última publicación de la Tabla (2000) y la fecha del estudio (2010), ha sido necesario aplicar la metodología RAS para actualizarla y obtener la TIO del turismo de la CV corres- 
pondiente a 2010. Por último, dado que el análisis era a nivel provincial, se ha procedido a regionalizar dicha Tabla utilizando la contabilidad regional disponible y nuevamente el RAS.

El método RAS permite recoger los cambios tecnológicos en el periodo que media entre la última TIO disponible y el año objeto de estudio, así como la regionalización de una TIO (de la TIO de CV a la TIO de la provincia de Valencia) ${ }^{2}$. La primera aplicación del RAS que se indica en la Figura 1 consiste en ajustar la matriz de relaciones intersectoriales de la CV al año 2010 a partir de la información suministrada por la TIO correspondiente al 2000 y los nuevos márgenes establecidos de los consumos y outputs intermedios obtenidos con la información de la contabilidad regional del INE. Por otra parte, sobre la TIO de la CV actualizada se vuelve a aplicar el RAS para obtener la Tabla de la provincia de Valencia, basándose en los márgenes de consumos y outputs intermedios referentes a Valencia. Todo ello da lugar a la obtención de la TIO del turismo de Valencia en 2010 (Tabla A1, A2, A3 del Anexo).

En el siguiente esquema se resumen las etapas seguidas en el análisis empírico.

Figura 1

Esquema metodológico del análisis input-output

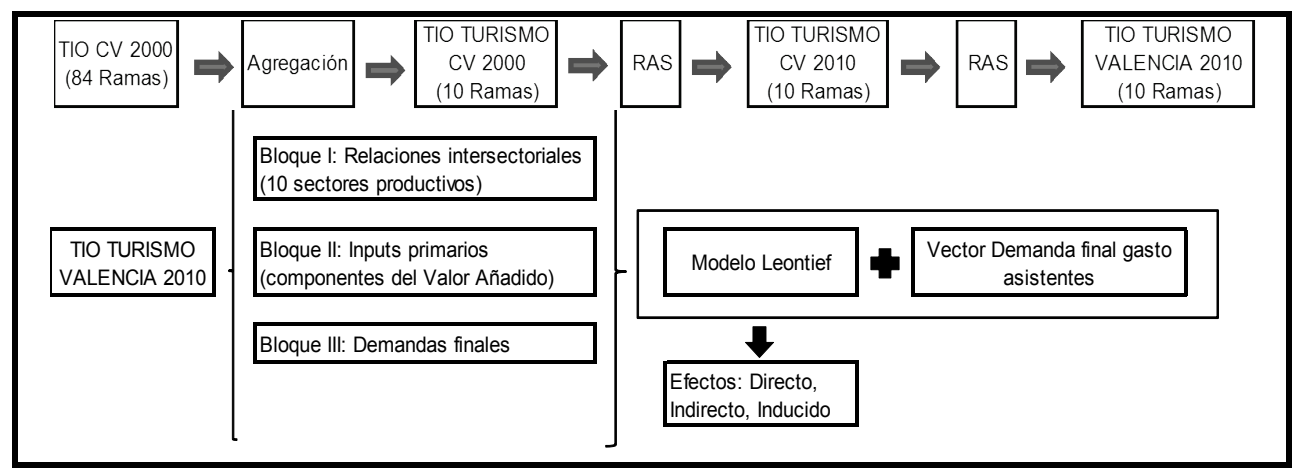

Fuente: Elaboración propia.

El método RAS $^{3}$ viene expresado a través de la operación matricial que le da el nombre (Pedreño, 1984):

${ }^{2}$ El método RAS ha sido considerado en economía desde hace años como el idóneo para la estimación de matrices Input-Output, encontrándose numerosas referencias sobre este procedimiento (Cabrer, et al., 2007).

${ }^{3}$ Ver Martí et al. (2009) para una explicación más detallada de la metodología aplicada 


$$
\mathrm{A}_{1}=\hat{\mathrm{R}} \times \mathrm{A}_{0} \times \hat{\mathrm{S}}
$$

donde,

$\mathrm{A}_{1}$ : matriz de transacciones estimada

$\hat{\mathrm{R}}$ y $\hat{\mathrm{S}}$ : matrices diagonales para la transformación de la matriz de partida $\mathrm{A}_{0}$

$\mathrm{A}_{0} \quad$ : matriz de transacciones original

En la práctica los vectores $\hat{\mathrm{R}}$ y $\hat{\mathrm{S}}$ se premultiplican y posmultiplican respectivamente, a la matriz de partida $\mathrm{A}_{0}$ proporcionando la convergencia de las filas y columnas agregadas de la matriz $\mathrm{A}_{1}$ hasta conseguir una matriz de transacciones actualizada.

Antes de proceder a aplicar esta técnica sobre la matriz intersectorial (Bloque I) es necesario realizar la actualización de los Bloques II y III de la TIO, correspondientes a los inputs primarios y a la demanda final. Para ello, una vez determinado el año de referencia (2010), se toma la máxima información contable disponible de las variables que componen dichas partes de la matriz: sueldos y salarios, seguridad social, valor añadido bruto, importaciones, exportaciones, entre otros. En ocasiones, y dado que dichos datos suelen ser simplemente un avance, no es posible utilizar toda la información porque no está completa y conduce a errores. Consecuentemente, se emplean todas aquellas variables que permitan reconstruir los inputs primarios y la demanda final verificando las identidades contables necesarias. Llegado a este punto se está en situación de proceder a aplicar la técnica RAS para el Bloque I, pues se dispone de toda la información de los Bloques II y III, así como los totales de las filas/columnas del Bloque I y, por tanto, tan sólo faltaría actualizar la matriz de relaciones intersectoriales del año $2000\left(\left[\mathrm{~T}_{\mathrm{ij}}\right]_{2000}\right)$ a las que se aplicaría de forma iterativa la ecuación (1). De forma detallada la aplicación del RAS sigue los siguientes pasos:

1. La primera iteración (ajuste de filas) comienza calculando el ratio $\mathrm{R}_{1}$ :

$$
R_{1}=\frac{\sum_{j=1}^{n} T_{i j}^{2010}}{\sum_{j=1}^{n} T_{i j}^{2000}}=\frac{b}{a_{1}} ; \mathrm{i}=1,2, \ldots, \mathrm{n} \text { sectores }
$$

2. Se diagonaliza el vector $\mathrm{R}_{1}$

$$
\widehat{R}_{1}=\left[\begin{array}{ccc}
r_{1} & \cdots & 0 \\
\vdots & \ddots & \vdots \\
0 & \cdots & r_{n}
\end{array}\right]
$$

3. Premultiplicamos el vector $\hat{R}_{1}$ por la matriz de transacciones $\left[\mathrm{T}_{\mathrm{ij}}\right]_{2000}$ reportando la nueva matriz $\left[\mathrm{T}_{\mathrm{ij}}\right]_{1}$

$$
\left[\hat{R}_{1}\right] \times\left[T_{i j}\right]_{2000}=\left[T_{i j}\right]_{1}
$$


Que servirá de base para iniciar la segunda iteración. Este ajuste que hemos proporcionado a las filas a través de la operación apuntada debemos llevarlo a cabo de igual forma para las columnas de la citada matriz.

4. Partiendo de la nueva matriz $\left[T_{i j}\right]_{1}$ definimos un nuevo ratio $\mathrm{S}_{1}$

$$
S_{1}=\frac{\sum_{j=1}^{n} T_{i j}^{2010}}{\sum_{j=1}^{n} T_{i j}^{1}} ; \mathrm{j}=(1,2, \ldots, \mathrm{n})
$$

5. Se diagonaliza el vector $\mathrm{S}_{1}$

$$
\hat{S}_{1}=\left[\begin{array}{ccc}
S_{1} & \cdots & 0 \\
\vdots & \ddots & \vdots \\
0 & \cdots & s_{n}
\end{array}\right]
$$

6. Con esta matriz diagonal a través de la segunda iteración y ajustando las columnas mediante:

$$
\left[T_{i j}\right]_{1} \times\left[\hat{S}_{1}\right]=\left[T_{i j}\right]_{2}
$$

De esta forma completamos la primera fase del ajuste biproporcional. El proceso continua haciendo $\mathrm{m}$-iteraciones hasta alcanzar una matriz $\left[T_{i j}\right]_{m}$ donde se debe de cumplir:

$$
\begin{aligned}
& \sum_{j=1}^{n} T_{i j}^{m}=\sum_{j=1}^{n} T_{i j}^{2010} \\
& \sum_{i=1}^{n} T_{i j}^{m}=\sum_{i=1}^{n} T_{i j}^{2010}
\end{aligned}
$$

Lo que implica que los vectores $\hat{R}_{m} y \hat{S}_{m}$ estimados en el proceso iterativo son idénticos a la matriz identidad.

La aplicación del modelo de Cantidades o de Leontief (1941) sobre la TIO representativa del turismo, permite cuantificar los efectos de retroalimentación surgidos en el sistema productivo a partir de una perturbación de la demanda final, como pueden ser los gastos realizados por los asistentes al Gran Premio. En definitiva, se fundamenta en la idea de que un incremento de la demanda se transmite, de modo que la producción no sólo aumenta en la cuantía necesaria para satisfacerla, sino en una superior para poder suministrar las demandas intermedias del resto de ramas y así alcanzar su incremento de producción. Se trata de una cadena de requerimientos sucesivos de inputs por parte de cada rama que debe aumentar su producción para abastecer a las demás.

Este modelo facilita la identificación de varios tipos de efectos de una actividad económica sobre el resto de las ramas y viceversa. Concretamente se pueden distinguir: efecto directo, efecto indirecto y efecto inducido. La definición de estos efectos aportada por la literatura no siempre es la misma, por ejemplo Seaman (2003) define el efecto directo como los gastos iniciales realizados en la celebración del evento analizado. Sin embargo, en el trabajo que presentamos se 
sigue la definición de Villaverde y Coto (1998), CEET (2005) y Gonzalez (2010) en donde se determinan los efectos a partir de un cálculo matricial. Por tanto, se ha considerado que:

- El efecto directo representa el esfuerzo productivo directo que deben realizar los agentes económicos del sector para hacer frente a una modificación de la demanda final de sus servicios.

- El efecto indirecto representa el impacto derivado de las sucesivas relaciones de compraventa que tienen lugar entre los sectores originariamente afectados por la actividad objeto de estudio y el resto de sectores económicos.

Generalmente son de menor magnitud que los directos porque surgen de las demandas generadas por los sectores que se han visto favorecidos en las primeras relaciones de compraventa (al incrementarse las compras de unos sectores, estos a su vez demandan de otros, y así sucesivamente generándose un entramado de relaciones intersectoriales).

- El efecto inducido es el generado por la capacidad de consumo e inversión de las empresas y agentes económicos directamente relacionados con la actividad de una rama. Para este cómputo se tienen que establecer supuestos complementarios sobre la capacidad de ahorro de las familias, asignando el resto a consumo final, y sobre la capacidad inversora, tanto del ahorro de las rentas del trabajo como del excedente bruto de explotación. Se trata, por tanto, del efecto multiplicador sobre la economía de los efectos directos e indirectos.

\section{RESULTADOS}

La aplicación de la metodología input-output permite conocer que efectos tiene sobre el sector turístico de la provincia de Valencia el gasto realizado por los asistentes al Gran Premio. Dentro de la información del Bloque II de la TIO de Valencia en 2010 destacan los sectores de "Actividades inmobiliarias" y "Restaurantes y similares" por ser los de mayor valor añadido a precios de mercado $\left(\mathrm{VAB}_{\mathrm{pm}}\right)$, mientras que las "Actividades Anexas al transporte" y las "Actividades inmobiliarias" destacan por sus consumos intermedios (Tabla 1). 
Tabla 1

Distribución sectorial del VAB y los consumos intermedios pertenecientes al sector turístico

\begin{tabular}{|c|c|c|}
\hline \multirow{2}{*}{ (miles de $€$ ) } & \\
\hline & VAB $_{p m}$ & Consumos intermedios \\
\hline Hoteles y similares & 314.904 & 10.739 \\
\hline Restaurantes y similares & 2.395 .074 & 97.679 \\
\hline Transporte de viajeros por ferrocarril & 232.521 & 6.071 \\
\hline Transporte de viajeros por carretera & 207.035 & 36.073 \\
\hline Transporte marítimo de viajeros & 2.740 & 10.375 \\
\hline Transporte aéreo de viajeros & 40.175 & 39.092 \\
\hline Actividades Anexas al transporte & 827.835 & 337.404 \\
\hline Actividades inmobiliarias & 5.624 .918 & 198.638 \\
\hline Alquiler de bienes de equipo de transporte & 694.931 & 59.359 \\
\hline Servicios culturales, recreativos y deportivos & 1.048 .838 & 542.134 \\
\hline
\end{tabular}

Nota: Consumos intermedios es la suma de las columnas correspondientes al bloque de relaciones intersectoriales.

Fuente: Elaboración propia.

En el caso del sector "Restaurantes y similares" el mayor VAB va ligado a una mayor concentración de empleo, aglutina más del $40 \%$ de los puestos de trabajo totales del turismo, mientras que en segundo lugar estarían los trabajadores de "Servicios culturales, recreativos y deportivos" representando un 16\% sobre el total (Gráfico 1). No obstante, se debe tener en cuenta que el empleo asociado a estas ramas no se explica sólo por el turismo, tal es el caso de los restaurantes o transporte terrestre donde se entremezcla la demanda de los propios residentes con la demanda realizada por los turistas.

\section{Gráfico 1}

Distribución sectorial del empleo en 2010 (personas)

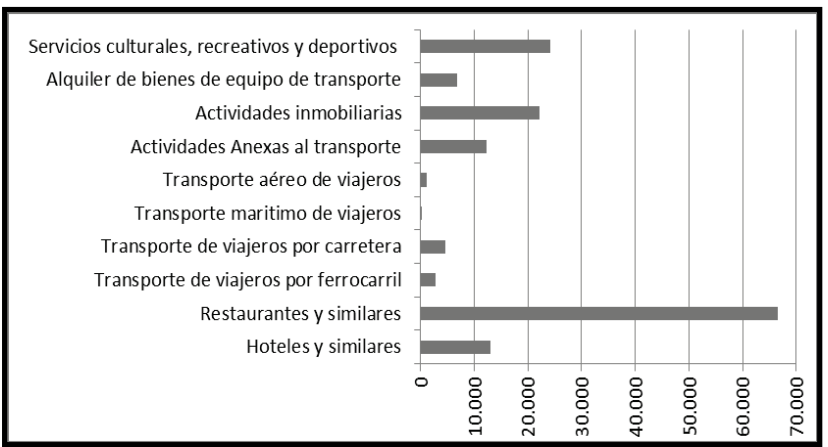

Fuente: Elaboración propia. 
El vector de demanda final utilizado en el análisis empírico ha sido el correspondiente al perfil del consumo de los asistentes al Campeonato Mundial de MotoGP. Todo ello ha requerido la especificación de cuáles son los gastos de los asistentes al evento según diferentes partidas. Se realizaron más de 200 encuestas donde se solicitaba información sobre el desembolso realizado en entradas, alojamiento, comidas, transporte, merchandising, ocio y otros, obteniendo la información recogida en la Tabla 2.

Tabla 2

Distribución del gasto realizado por los espectadores

\begin{tabular}{|l|r|c|}
\hline \multicolumn{1}{|c|}{ Partidas } & \multicolumn{1}{c|}{$\begin{array}{c}\text { Gasto Total } \\
\text { (euros) }\end{array}$} & $\begin{array}{c}\text { Gasto medio } \\
\text { (euros) }\end{array}$ \\
\hline Entrada & $5.106 .022,37$ & 76,20 \\
\hline Alojamiento & $3.445 .304,41$ & 104,59 \\
\hline Comidas & $3.277 .890,15$ & 45,12 \\
\hline Transporte Carretera & $1.807 .713,09$ & 30,82 \\
\hline Transporte ferrocarril & $36.325,74$ & 23,00 \\
\hline Transporte Aéreo & $1.960 .687,32$ & 395,00 \\
\hline Merchandising & $1.946 .021,65$ & 40,30 \\
\hline Ocio & $1.416 .929,39$ & 62,80 \\
\hline Otros gastos & $606.933,13$ & 46,38 \\
\hline
\end{tabular}

Fuente: Elaboración propia.

Dadas las características propias del evento, los asistentes fueron en su mayoría hombres, en torno al $70 \%$. Además, presentaban un perfil caracterizado por tener una renta media, una edad comprendida entre 18 y 70 años (con una media de 34 años) y ser un trabajador especializado, autónomo o estudiante.

Según la clasificación de los gastos descritos en la Tabla 2, la partida de mayor cuantía es evidentemente el transporte aéreo, no obstante tan solo lo utilizó el $6 \%$. Se trata de turistas internacionales procedentes de Europa (Francia, Italia, Alemania, Reino Unido y Bélgica) y del continente americano (Argentina, Brasil, México, Venezuela y EEUU). Por otra parte, los españoles (no residentes en Valencia) que acudieron al evento representaron el $40 \%$ y utilizaron el coche o el tren, desembolsando por término medio 30,82 euros y 23 euros respectivamente ${ }^{4}$. De manera que más de la mitad de los asistentes eran procedentes de la CV.

\footnotetext{
${ }^{4}$ Se debe tener en cuenta que los gastos de transporte han sido imputados en su totalidad a Valencia, pues no se disponía de información para discernir entre los realizados en la provincia y fuera de ella.
} 
Por otra parte, un $17 \%$ entraron con invitación, mientras que el resto pagó entre 900 y 22 euros, siendo la media de 76,2 euros. Respecto al gasto en pernoctación hay que señalar que tan solo utilizaron alojamiento externo el $40 \%$ de los asistentes. El más elegido fue el hotel de 2 o 3 estrellas (16,5\%), en segundo lugar se encuentran los que se alojaron en un camping (11\%) y otros optaron por el lujo del hotel de 4 o 5 estrellas (7\%), el resto se distribuyó entre hostales o acampada libre.

Los gastos en merchandising hacen referencia a la compra de camisetas, gorras, pañuelos, etc. diseñados exclusivamente para el evento, siendo sus consumidores el $60 \%$ de los aficionados que acudieron al campeonato. Las partidas de ocio se concretan en entradas de cine, museos, discotecas que realizan los aficionados al terminar las distintas pruebas, permitiendo visitar los lugares más emblemáticos de Valencia. Por término medio estos gastos ascendieron a 62,8 euros.

Aplicando el modelo de Cantidades de Leontief se han obtenido los resultados del impacto económico concretados en las siguientes macromagnitudes ${ }^{5}$ : Salarios, beneficios, ingresos fiscales, PIB, producción y empleos, diferenciando entre su efecto directo, indirecto e inducido (Tabla 3). Dado el objetivo del artículo, y al igual que Tarancón (2005) y Lee y Taylor (2005), todos los resultados se centran en las ramas de actividad características del sector turístico (Tabla 3 y 4), por tanto las conclusiones obtenidas están mermadas al no tener en cuenta el efecto arrastre en el resto de sectores de la economía.

Tabla 3

Impacto económico del gasto de los asistentes al Campeonato Mundial de MotoGP

\begin{tabular}{|l|r|r|r|r|c|}
\cline { 2 - 7 } \multicolumn{1}{c|}{ (miles de $\boldsymbol{\epsilon})$} & Directo & Indirecto & Inducido & Totales & $\%$ s/VAL \\
\hline Salarios & 7.438 & 278 & 3.191 & 10.907 & $\mathbf{0 , 3 1 4 \%}$ \\
\hline Beneficios empresariales & 8.317 & 391 & 7.383 & 16.090 & $\mathbf{0 , 2 1 0 \%}$ \\
\hline Ingresos Fiscales & 18 & 16 & 221 & 256 & $\mathbf{0 , 1 0 1 \%}$ \\
\hline PIB & 15.694 & 685 & 10.697 & 27.077 & $\mathbf{0 , 2 4 0 \%}$ \\
\hline Producción total & 22.633 & 1.014 & 12.484 & 36.132 & $\mathbf{0 , 2 6 3 \%}$ \\
\hline Empleos (personas) & 421 & 13 & 142 & 575 & $\mathbf{0 , 3 7 4 \%}$ \\
\hline
\end{tabular}

Fuente: Elaboración propia.

Según los resultados del impacto económico, el efecto directo es el más significativo, es decir, el gasto realizado por los asistentes ha tenido una repercusión directa sobre el resto de sectores, incrementando su demanda y como

\footnotetext{
${ }^{5}$ En las tablas del anexo A4 y A5 se aporta la desagregación sectorial de los efectos sobre el PIB y el empleo.
} 
consecuencia el PIB en más de 15 millones de euros. Además se observa que la distribución del PIB entre salarios y beneficios es muy similar. En el caso de los ingresos fiscales el impacto se ve mermado por la concesión de subvenciones al sector transporte, dando lugar a unos impuestos indirectos de 78.000 euros que se ven reducidos por unas subvenciones de 60.000 euros. Por último, destacar que la actividad de los aficionados ha dado lugar a la creación de 421 empleos, consecuencia del incremento directo de la demanda generada en el sector.

Respecto al efecto indirecto se debe resaltar que, en su conjunto, los turistas han contribuido a aumentar el PIB en 685.000 euros distribuyéndose un $40 \%$ en salarios y un $57 \%$ en beneficios empresariales. Esto significa que las segundas o terceras relaciones intersectoriales tienen un suave impacto en la economía, aunque en ningún caso despreciable.

El impacto inducido requiere establecer una hipótesis sobre la repercusión en el consumo y la inversión de las rentas generadas. En este estudio se ha considerado que un $70 \%$ de las mismas se han destinado a atender el gasto interno, dando lugar a nuevas demandas en el resto de sectores que incrementa la actividad turística. Concretamente se ha generado una producción total de más de 12 millones de euros, siendo una cifra muy superior a la alcanzada por el efecto indirecto.

La cuarta columna de la Tabla 3 muestra el efecto total comparándose en la quinta columna con el volumen que el sector turístico representa en toda la provincia de Valencia. Así, se concluye que la celebración del Campeonato aporta el $0,3 \%$ del total de los salarios y empleos que el turismo genera en Valencia y el $0,24 \%$ del PIB. Manifestando su grado de importancia dentro de la actividad turística valenciana.

El desglose de los efectos a nivel sectorial es una de las ventajas que aporta la metodología input-output, permitiendo enriquecer los resultados del análisis. Con objeto de determinar qué sectores turísticos recogen el mayor impacto, a continuación se muestran los resultados desagregados sectorialmente (Tabla 4).

Haciendo un análisis comparativo, en términos absolutos, en el efecto directo de los 10 sectores considerados, los "Servicios culturales, recreativos y deportivos" destaca significativamente frente al resto, representando el 57\%. Esto tiene cierta lógica puesto que las partidas que mayor peso tienen en el desembolso inicial (entradas y ocio) pertenecen a este sector.

El efecto indirecto, de forma generalizada, pierde valor frente al directo, 1legando a ser nulo en algunos de los sectores relacionados con el transporte (Tabla 4). Todo ello puede deberse al ámbito geográfico del estudio y la escasez de segundas y terceras relaciones de compraventa intersectoriales necesarias para cubrir las necesidades de los espectadores. No obstante, es nuevamente el sector "Servicios culturales, recreativos y deportivos" el de mayor impacto, 
representando un $64,15 \%$ sobre el total el efecto indirecto que constituyen las actividades del turismo.

Tabla 4

Distribución sectorial del impacto económico del gasto de los asistentes al Campeonato Mundial de MotoGP sobre los consumos intermedios

\begin{tabular}{|c|c|c|c|}
\hline (miles de $€$ ) & Directo & Indirecto & Inducido \\
\hline Hoteles y similares & 35 & 8 & 59 \\
\hline Restaurantes y similares & 238 & 3 & 17 \\
\hline Transporte de viajeros por ferrocarril & 1 & 0 & 1 \\
\hline Transporte de viajeros por carretera & 229 & 3 & 34 \\
\hline Transporte marítimo de viajeros & 5 & 0 & 9 \\
\hline Transporte aéreo de viajeros & 24 & 0 & 2 \\
\hline Actividades Anexas al transporte & 248 & 23 & 121 \\
\hline Actividades inmobiliarias & 515 & 25 & 345 \\
\hline Alquiler de bienes de equipo de transporte & 600 & 23 & 79 \\
\hline Servicios culturales, recreativos y deportivos & 2.518 & 153 & 352 \\
\hline
\end{tabular}

Fuente: Elaboración propia.

Tal y como se observa en la Tabla 4 el incremento de las rentas (efecto inducido) da lugar a un aumento de los consumos intermedios en las 10 ramas de la actividad turística, aunque a unos niveles inferiores que el efecto directo. En este caso las "Actividades inmobiliarias" llegan a niveles semejantes a los "Servicios culturales, recreativos y deportivos". En definitiva, las actividades inmobiliarias han sido las más beneficiadas por el efecto arrastre provocado por el gasto de los asistentes al Campeonato, pues la información de partida no recoge ninguna dedicación monetaria a este sector por parte de los aficionados.

\section{CONCLUSIONES}

En el trabajo realizado se ha analizado el impacto económico que la celebración de un evento deportivo de relevancia internacional como es el Campeonato de MotoGP genera sobre el turismo valenciano. La utilización del análisis inputoutput ha permitido diferenciar entre los efectos directamente aflorados como consecuencia del incremento de demanda provocado por el gasto que los asistentes realizan, de las segundas interrelaciones producidas en el entramado del sector turístico en la provincia de Valencia. Igualmente, las rentas suscitadas de todas estas relaciones van a repercutir nuevamente en la economía en una cuantía significativa.

Los resultados presentados han incluido la repercusión que el gasto de los 80.000 aficionados provoca en el sector turístico. No cabe duda que la celebra- 
ción de una competición de estas características, va a tener siempre repercusiones positivas en el lugar donde se celebre. Como avala el estudio, este Campeonato deportivo genera ingresos fiscales por un valor superior de 256.000 euros, así como un incremento del PIB de más de 27 millones de euros representando el $0,24 \%$ de toda la producción del turismo valenciano, sin olvidar el corto lapso de tiempo que dura dicha celebración. Es importante considerar que el impacto obtenido se limita a la provincia de Valencia sin tener en consideración las importaciones de inputs requeridos para proveer estos servicios.

Además de los efectos económicos, en ningún caso cuestionables, la construcción del Circuit Ricardo Tormo ha supuesto una notable mejora de infraestructuras y accesos de los que todos los ciudadanos pueden beneficiarse $\mathrm{y}$, en ocasiones, no valorados. Por otra parte, es importante tener presente, que la celebración de dicho evento conlleva una serie de gastos de organización y mantenimiento de las instalaciones que deberían tenerse en cuenta con objeto de determinar la viabilidad del mismo. No obstante, la imposibilidad de acceder a dicha información, ha impedido su inclusión en el trabajo.

El empleo de dinero público para la realización de eventos deportivos tendría que ir siempre acompañado de un estudio de impacto económico, con objeto de determinar si los posibles beneficios económicos y sociales de dicha competición justifican la inversión propuesta. Igualmente, habría que valorar no sólo el periodo de celebración del Campeonato, sino también el alcance internacional del mismo. Las retrasmisiones televisivas internacionales suponen un "escenario" de incalculable valía.

Por tanto, puede llegarse a la conclusión que los eventos deportivos, además de provocar un crecimiento económico, son el escenario perfecto para dar a conocer la riqueza y cultura en la zona donde se celebran.

\section{REFERENCIAS BIBLIOGRÁFICAS}

ALONSO, JC.; RUESGAS, S. y SAEZ, F. (1991). "Impacto económico del deporte en España" en Revista de Investigaciones y Documentación sobre las Ciencias de la Educación Física y el deporte, 18 pp. 22-35.

BARGUET, E. y GOUGUET, J.J. (2008). "Un análisis de la Copa del Mundo de Rugby 2007 en Francia" en Revista Asturiana de Economía, 42, pp. 103127.

BARKER, M.; PAGE, S.J. y MEYER, D. (2002). "Evaluating the impact of the 2000 America's Cup on Auckland, New Zealand"en Event Management, 7 (2), pp. 79-92. 
BRACALENTE, B.; CHIRIELEISON, C.; COSSIGNANI, M.; FERRUCCI, L.; GIGLIOTTI, M. y RANALLI, M (2011). "The economic impact of cultural events: the Umbria Jazz Music Festival" en Tourism Economics, 17 (6), pp. 1235-1255.

BRUNET, F. (1992). Economía de los Juegos Olímpicos Barcelona'92, BarceIona

CABRER, B.; OLMOS, J.; PAVIA, J.M. y SALA, R. (2007). "Actualización de matrices origen-destino. Un análisis de alternativas a través de MonteCarlo" en XV Jornadas de ASEPUMA.

CEET (2005). Impacto económico de los juegos olímpicos Madrid 2012. publicación del Centro de Estudios Económicos Tomillo junto el Ayuntamiento de Madrid.

COLLINS, A.; JONES, C. y MUNDAY, M. (2009). "Assessing the environmental impacts of mega sporting events: Two options?" en Tourism Management, 30, pp. 828-837.

CROMPTON, J.; LEE, S. y SHUSTER, T.J. (2001). "A guide for undertaking economic impact studies: The Springfest example" en Journal of Travel Resarch, 40, pp. 79-87.

ESPEJO-SAAVEDRA, J.L. y ALGARRA, A.A. (2005). "Elaboración de un modelo de impacto del fútbol profesional sobre la economía española" en $I$ Jornadas de Análisis input-output, Oviedo.

FERNÁNDEZ, JI.; MARTí, ML. y PUERTAS, R. (2007). "Análisis del impacto económico de la Copa América" en Congreso de Economía Aplicada.

FOMENTO DE SAN SEBASTIAN (2005). Impacto económico del XVI campeonato del mundo de atletismo master stadia. www.fomentosansebastian.org

FOURIE, J. y SANTANA-GALLEGO, M. (2011). "The impact of mega-sport events on tourist arrivals" en Tourism Management, 32 pp. 1364-1370.

GONZALEZ, M. (2010). "Impacto económico de los hoteles: Aplicación a la ciudad de Sevilla", en PASOS. Revista de Turismo y Patrimonio Cultural, 2, pp. 319-338.

HERDERSON, P.H. (1955). "El método del factor-producto: Una aplicación del mismo a la economía italiana", en Moneda y Crédito, 54, pp.3-25.

HILL, J. (1975). The economic impact of the Port of Baltimore and Maryland. Division of transport, business and public policy. College of Business and Management. University of Maryland.

KASIMATI, E. (2003). "Economic aspects and the summer Olympics: a review of related research", en International Journal of Tourist Research, 5 (6), pp. 433- 444.

LEE, C. y TAYLOR, T. (2005). "Critical reflections on the economic impact assessment of a mega-event: the case of 2002 FIFA World Cup" en Tourism Management, 26, pp. 595-603. 
LEONTIEF, W. (1941). The Structure of American Economy, 1919-1929: an Empirical Application of Equilibrium Analysis, Cambridge, Harvard University Press.

LOPEZ, L.A.; MONSALVE, F y ZAFRILLA, J.E. (2011). "Análisis Input-Output de la eficacia de la Política Europea de desarrollo rural 2007-2013. Propuesta metodológica y resultados para Castilla-La Mancha" en Estudios de Economía Aplicada, 29, pp. 223-246.

MARTÍ, ML.; PUERTAS, R. y FERNÁNDEZ, J.I. (2009). Metodología para el análisis de impacto portuario: Aplicación a los puertos de Gandía, Sagunto y Valencia. Fundación Valenciaport.

MARTINEZ DEL CASTILLO, J.; PUIG, N. y FRAILE, A. (1991). "La estructura ocupacional del deporte en España". Encuesta realizada sobre sectores de entrenamiento, docencia, animación y dirección, MEC-CSD. Madrid.

MAUDOS, J. (2007). "Impacto económico de la $32^{\text {a }}$ America's Cup Valencia 2007" Informe final IVIE.

PEDREÑO, A. (1984). "Algunas reflexiones en torno al método RAS como técnica de ajuste de la matriz de flujos intersectoriales" en Revista de Economía y Empresa, 2, pp. 51-67

PEDROSA, R. y SALVADOR, J.A. (2003). "El impacto del deporte en la economía: problemas de medición" en Revista Asturiana de Economía, 26, pp. 61-84

PERLES, J.F. (2006). "Análisis del impacto económico de eventos: una aplicación a fiestas populares de proyección turística" en Cuadernos de Turismo, 17, pp. 147-166.

POLO, C. y VALLE, E. (2011). "El peso del turismo en las islas Baleares: 19831997-2004" en Estudios de Economia Aplicada, 29, pp. 737-754.

QUESADA, S. y DIEZ M.D. (1993). "Economía pública del deporte no profesional" en VII Reunión Anual ASEPELT-España, Cádiz, 17-18 junio.

SEAMAN, B. (2003). "Economic impact of the arts" en TOWSE R. (2003) A Ahandbook of Cultural Economics, 27, pp.224-231.

STONE, R. (1969). "L'analisi dei Sistema Economici" en D’Adda, C., y Filippini, C.: Interdependenze Industriali e Politica Economica, I1 Mulino, Bologna.

TARANCON, M.A. (2005). "Diferencias estructurales en las actividades del turismo entre Andalucía y Comunidad Valenciana: aplicación del análisis input-output cualitativo con análisis del sensibilidad" en Revista de Estudios Regionales, 72, pp. 165-189.

VILLAVERDE, J. y COTO, P. (1998). "Port economic impact, methodologies and application to the Port of Santander" en International Journal of Transport Economics, 25, pp. 159-179.

WATSON, P.; DAVIES, S. y THILMANY, D. (2008). "Determining economic contributions in a recreational industry. An application to Colorado's Golf Industry" en Journal of Sport Economic, 9 (6), pp. 571-591. 


\section{ANEXO}

Tabla A1

Bloque I de los sectores del turismo pertenecientes a la Tabla Input-Output de Valencia para el 2010

(miles de $€$ )

\begin{tabular}{|c|c|c|c|c|c|c|c|c|c|c|}
\hline $\begin{array}{l}\text { Relaciones } \\
\text { Intersectoriales del } \\
\text { turismo }\end{array}$ & $\begin{array}{c}\text { Hoteles } \\
y \\
\text { similares }\end{array}$ & $\begin{array}{c}\text { Restaurantes } \\
\text { y similares }\end{array}$ & $\begin{array}{l}\text { Transporte } \\
\text { de viajeros } \\
\text { ferrocarril }\end{array}$ & $\begin{array}{c}\text { Transporte } \\
\text { de viajeros } \\
\text { carretera }\end{array}$ & $\begin{array}{c}\text { Transporte } \\
\text { marítimo } \\
\text { de viajeros }\end{array}$ & $\begin{array}{c}\text { Transporte } \\
\text { aéreo de } \\
\text { viajeros }\end{array}$ & $\begin{array}{l}\text { Actividades } \\
\text { Anexas al } \\
\text { transporte }\end{array}$ & $\begin{array}{c}\text { Actividades } \\
\text { inmobiliarias }\end{array}$ & $\begin{array}{c}\text { Alquiler } \\
\text { de } \\
\text { bienes de } \\
\text { equipo de } \\
\text { transporte }\end{array}$ & $\begin{array}{c}\text { Servicios } \\
\text { culturales, } \\
\text { recreativos } \\
y \\
\text { deportivos }\end{array}$ \\
\hline Hoteles y similares & 1.816 & 728 & 1.379 & 54 & 0 & 0 & 135.798 & 0 & 0 & 0 \\
\hline $\begin{array}{l}\text { Restaurantes y } \\
\text { similares }\end{array}$ & 579 & 8.821 & 478 & 82 & 10 & 14.378 & 612 & 0 & 24 & 6.556 \\
\hline $\begin{array}{l}\text { Transporte de } \\
\text { viajeros por ferrocarril }\end{array}$ & 20 & 192 & 195 & 0 & 0 & 0 & 902 & 0 & 0 & 0 \\
\hline $\begin{array}{l}\text { Transporte de } \\
\text { viajeros por carretera }\end{array}$ & 48 & 0 & 1.939 & 29.849 & 0 & 23 & 1.437 & 0 & 318 & 0 \\
\hline $\begin{array}{l}\text { Transporte marítimo } \\
\text { de viajeros }\end{array}$ & 40 & 1.778 & 0 & 128 & 6.076 & 0 & 2.794 & 0 & 0 & 0 \\
\hline $\begin{array}{l}\text { Transporte aéreo } \\
\text { de viajeros }\end{array}$ & 0 & 2 & 0 & 0 & 0 & 1.860 & 4.101 & 0 & 2 & 0 \\
\hline $\begin{array}{l}\text { Actividades Anexas } \\
\text { al transporte }\end{array}$ & 868 & 2.571 & 1.696 & 5.054 & 3.953 & 0 & 181.060 & 1.603 & 11.179 & 27.523 \\
\hline Actividades inmobiliarias & 6.738 & 81.241 & 17 & 495 & 2 & 287 & 5.932 & 197.035 & 5.567 & 50.829 \\
\hline $\begin{array}{l}\text { Alquiler de bienes de } \\
\text { equipo de transporte }\end{array}$ & 97 & 2.347 & 365 & 407 & 334 & 22.541 & 4.765 & 0 & 42.255 & 47.009 \\
\hline $\begin{array}{l}\text { Servicios culturales, } \\
\text { recreativos, deportivos }\end{array}$ & 532 & 0 & 3 & 3 & 0 & 2 & 4 & 0 & 14 & 410.216 \\
\hline
\end{tabular}

Fuente: Elaboración propia.

Tabla A2

Bloque II de los sectores del turismo pertenecientes a la Tabla Input-Output de Valencia para el 2010

(miles de $€$ )

\begin{tabular}{|c|c|c|c|c|c|c|c|c|c|c|}
\hline $\begin{array}{c}\text { Inputs primarios del } \\
\text { turismo }\end{array}$ & $\begin{array}{c}\text { Hoteles } \\
y \\
\text { similares }\end{array}$ & $\begin{array}{c}\text { Restaurantes } \\
\text { y similares }\end{array}$ & \begin{tabular}{|l} 
Transporte \\
de viajeros \\
ferrocarril
\end{tabular} & $\begin{array}{c}\text { Transporte } \\
\text { de viajeros } \\
\text { carretera }\end{array}$ & $\begin{array}{c}\text { Transporte } \\
\text { marítimo } \\
\text { de } \\
\text { viajeros }\end{array}$ & $\begin{array}{l}\text { Transporte } \\
\text { aéreo de } \\
\text { viajeros }\end{array}$ & $\begin{array}{c}\text { Actividades } \\
\text { Anexas } \\
\text { al trans- } \\
\text { porte }\end{array}$ & $\begin{array}{c}\text { Actividades } \\
\text { inmobiliarias }\end{array}$ & \begin{tabular}{|} 
Alquiler \\
de \\
bienes de \\
equipo de \\
transporte
\end{tabular} & $\begin{array}{c}\text { Servicios } \\
\text { culturales, } \\
\text { recreativos y } \\
\text { deportivos }\end{array}$ \\
\hline Valor Añadido bruto apm & 314.904 & 2.395 .074 & 232.521 & 207.035 & 2.740 & 40.175 & 827.835 & 5.624 .918 & 694.931 & 1.048 .838 \\
\hline Total importaciones & 40.856 & 3.222 & 150.806 & 20.333 & 39.137 & 80.156 & 321.592 & 64.220 & 156.823 & 123.216 \\
\hline
\end{tabular}

Fuente: Elaboración propia. 
Tabla A3

Bloque III de los sectores del turismo pertenecientes a la Tabla Input-Output de Valencia para el 2010

(miles de €)

\begin{tabular}{|l|r|r|}
\cline { 2 - 3 } \multicolumn{1}{c|}{} & Demanda final & Total empleos \\
\hline Hoteles y similares & 226.725 & 366.499 \\
\hline Restaurantes y similares & 2.464 .437 & 2.495 .975 \\
\hline Transporte de viajeros por ferrocarril & 388.089 & 389.397 \\
\hline Transporte de viajeros por carretera & 229.827 & 263.441 \\
\hline Transporte marítimo de viajeros & 41.435 & 52.252 \\
\hline Transporte aéreo de viajeros & 153.457 & 159.423 \\
\hline Actividades Anexas al transporte & 1.251 .323 & 1.486 .831 \\
\hline Actividades inmobiliarias & 5.539 .633 & 5.887 .776 \\
\hline Alquiler de bienes de equipo de transporte & 790.993 & 911.113 \\
\hline Servicios culturales, recreativos, deportivos & 1.303 .413 & 1.714 .188 \\
\hline
\end{tabular}

Fuente: Elaboración propia.

Tabla A4

Desagregación sectorial del impacto económico sobre el PIB

\begin{tabular}{|l|r|r|r|}
\cline { 2 - 4 } \multicolumn{1}{c|}{ (miles de $\boldsymbol{\epsilon}$ ) } & Directo & Indirecto & Inducido \\
\hline Hoteles y similares & 2.944 & 21 & 253 \\
\hline Restaurantes y similares & 3.308 & 13 & 2.462 \\
\hline Transporte de viajeros por ferrocarril & 22 & 0 & 230 \\
\hline Transporte de viajeros por carretera & 1.505 & 20 & 206 \\
\hline Transporte marítimo de viajeros & 0 & 0 & 6 \\
\hline Transporte aéreo de viajeros & 505 & 0 & 3 \\
\hline Actividades Anexas al transporte & 100 & 49 & 330 \\
\hline Actividades inmobiliarias & 406 & 106 & 5.793 \\
\hline Alquiler de bienes de equipo de transporte & 384 & 80 & 503 \\
\hline Servicios culturales, recreativos, deportivos & 6.520 & 397 & 912 \\
\hline Total & 15.694 & 685 & 10.697 \\
\hline
\end{tabular}

Fuente: Elaboración propia. 


\section{Tabla A5}

Desagregación sectorial del impacto económico sobre el empleo

\begin{tabular}{|l|r|r|c|}
\cline { 2 - 4 } \multicolumn{1}{c|}{ (miles de $\boldsymbol{\epsilon})$} & Directo & Indirecto & Inducido \\
\hline Hoteles y similares & 123 & 1 & 11 \\
\hline Restaurantes y similares & 94 & 0 & 70 \\
\hline Transporte de viajeros por ferrocarril & 0 & 0 & 3 \\
\hline Transporte de viajeros por carretera & 35 & 0 & 5 \\
\hline Transporte marítimo de viajeros & 0 & 0 & 0 \\
\hline Transporte aéreo de viajeros & 14 & 0 & 0 \\
\hline Actividades Anexas al transporte & 2 & 1 & 5 \\
\hline Actividades inmobiliarias & 2 & 0 & 23 \\
\hline Alquiler de bienes de equipo de transporte & 4 & 1 & 5 \\
\hline Servicios culturales, recreativos, deportivos & 148 & 9 & 21 \\
\hline Total & $\mathbf{4 2 1}$ & $\mathbf{1 3}$ & $\mathbf{1 4 2}$ \\
\hline
\end{tabular}

Fuente: Elaboración propia. 